\title{
Thermoelectric study of crossroads material MnTe via sulfur doping
}

\author{
Wenjie Xie, ${ }^{1, a)}$ Sascha Populoh, ${ }^{1}$ Krzysztof Gałaz zka,,${ }^{1,2}$ Xingxing Xiao, ${ }^{3}$ Leyre Sagarna, ${ }^{1}$ \\ Yufei Liu, ${ }^{4}$ Matthias Trottmann, ${ }^{1}$ Jian He, ${ }^{4}$ and Anke Weidenkaff ${ }^{1,3}$ \\ ${ }^{1}$ Empa-Swiss Federal Laboratories for Materials Science and Technology, Solid State Chemistry and \\ Catalysis, Uberlandstrasse 129, CH-8600 Dübendorf, Switzerland \\ ${ }^{2}$ Department of Chemistry and Biochemistry, University of Bern, Freiestrasse 3, CH-3012 Bern, Switzerland \\ ${ }^{3}$ Institute for Materials Science, University of Stuttgart, DE-70569 Stuttgart, Germany \\ ${ }^{4}$ Department of Physics and Astronomy, Clemson University, Clemson, South Carolina 29634-0978, USA
}

(Received 28 January 2014; accepted 4 March 2014; published online 14 March 2014)

\begin{abstract}
Here, we report thermoelectric study of crossroads material MnTe via iso-electronic doping $\mathrm{S}$ on the Te-site. $\mathrm{MnTe}_{1-\mathrm{x}} \mathrm{S}_{\mathrm{x}}$ samples with nominal $\mathrm{S}$ content of $\mathrm{x}=0.00,0.05$, and 0.10 were prepared using a melt-quench method followed by pulverization and spark plasma sintering. The X-ray powder diffraction, scanning electron microscopy, and ZAF-corrected compositional analysis confirmed that $\mathrm{S}$ uniformly substitutes Te up to slightly over $2 \%$. A higher content of $\mathrm{S}$ in the starting materials led to the formation of secondary phases. The thermoelectric properties of $\mathrm{MnTe}_{1-\mathrm{x}} \mathrm{S}_{\mathrm{x}}$ samples were characterized by means of Seebeck coefficient, electrical conductivity, and thermal conductivity measurements from $300 \mathrm{~K}$ to $773 \mathrm{~K}$. Furthermore, Hall coefficient measurements and a single parabolic band model were used to help gain insights on the effects of S-doping on the scattering mechanism and the carrier effective mass. As expected, $\mathrm{S}$ doping not only introduced hole charge carriers but also created short-range defects that effectively scatter heat-carrying phonons at elevated temperatures. On the other hand, we found that $\mathrm{S}$ doping degraded the effective mass. As a result, the $Z T$ of $\mathrm{MnTe}_{0.9} \mathrm{~S}_{0.1}$ was substantially enhanced over the pristine sample near $400 \mathrm{~K}$, while the improvement of $Z T$ became marginal at elevated temperatures. A $Z T \sim 0.65$ at $773 \mathrm{~K}$ was obtained in all three samples. (C) 2014 AIP Publishing LLC. [http://dx.doi.org/10.1063/1.4868584]
\end{abstract}

\section{INTRODUCTION}

Over the past two decades, there has been heightened interest in developing novel high performance thermoelectric (TE) materials for environmentally friendly, efficient power generation and solid-state refrigeration. ${ }^{1-5}$ The performance of TE material is gauged by its figure of merit, $Z T=\sigma \alpha^{2} T / \kappa$, where $\sigma$ is the electrical conductivity, $\alpha$ the Seebeck coefficient, $\kappa$ the total thermal conductivity, and $T$ the absolute temperature. The state-of-the-art TE materials have a maximum $Z T \sim 1-2$ because $\sigma, \alpha$, and $\kappa$ are mutually counterindicated: optimizing one property often adversely affects other properties. For decades, tellurides such as $\mathrm{Bi}_{2} \mathrm{Te}_{3}$ and $\mathrm{PbTe}$ have been constituted the cornerstone of the state-of-the-art TE materials. An immediate question arises as to whether one can find novel, higher performance, and environmentally friendlier TE tellurides. To this end, we herein report a thermoelectric study of sulfur-doped manganese telluride $(\mathrm{Mn}(\mathrm{Te}, \mathrm{S}))$.

MnTe is known as a crossroads material in view of the other manganese chalcogenides $\mathrm{MnX}(\mathrm{X}=\mathrm{O}, \mathrm{S}$, and $\mathrm{Se})$ : (i) MnTe adopts a hexagonal NiAs structure in its ground state, whereas $\mathrm{MnX}$ adopts a cubic $\mathrm{NaCl}$ crystal structure; and (ii) MnTe possesses a resistivity at least 3 orders of magnitude lower than other MnX compounds. While most known Mncontaining binary compounds are either insulating $(\mathrm{MnO}$, $\mathrm{MnS}$, and $\mathrm{MnSe}$ ) or metallic (MnSb and MnAs), MnTe is

\footnotetext{
a) Author to whom correspondence should be addressed. Electronic mail: xie@imw.uni-stuttgart.de. Present address: Institute for Materials Science, University of Stuttgart, DE-70569 Stuttgart, Germany.
}

one of a few known to be semiconducting with an optical band gap of $\sim 1.27 \mathrm{eV}^{6-8}$ and a thermal band gap of $\sim 0.86 \mathrm{eV}$. ${ }^{9}$ We know that semiconductors with band gap $\Delta \sim 10 k_{\mathrm{B}} T_{\mathrm{o}}$ make good thermoelectrics, $k_{\mathrm{B}}$ is the Boltzmann constant, and $T_{\mathrm{o}}$ the operating temperature. Such a band gap size ensures a reasonable electrical conductivity without provoking the detrimental bipolar effect on the Seebeck coefficient. ${ }^{10}$ This " $10 k_{\mathrm{B}} T_{\mathrm{o}}$ " rule holds for either a direct or an indirect gap, and for both phonon and impurity scattering mechanisms. ${ }^{10}$ In addition, the high-symmetry crystal structure of MnTe is thermoelectrically favorable as it promotes electronic band degeneracy for high Seebeck coefficient. In these regards, MnTe is a promising material for TE power generation in the intermediate temperature range.

On the other hand, MnTe has features that are thermoelectrically unfavorable. Notably, the electronegativity difference between $\mathrm{Mn}$ and $\mathrm{Te}$ is substantially larger than those of the state-of-the-art TE tellurides such as $\mathrm{Bi}_{2} \mathrm{Te}_{3}$ and PbTe. The resulting stronger optical phonon is detrimental to the carrier mobility. In addition, the strong bonding and relatively light constituent element weight imply undesired high lattice thermal conductivity. In these regards, MnTe is reminiscent of oxides. ${ }^{11}$

The previous study of MnTe is mainly on its lowtemperature structural, magnetic, electrical, and optical properties, ${ }^{12-18}$ with little attention having been paid to its potential in thermoelectricity. Until recently, it was reported that the room temperature Seebeck coefficient of pristine MnTe $\alpha>600 \mu \mathrm{V} / \mathrm{K},{ }^{19}$ more than twice higher than $\mathrm{Bi}_{2} \mathrm{Te}_{3}{ }^{20,21}$ The conduction type is $p$-type in pristine MnTe, indicating holes are the majority carriers. Such a high 
Seebeck coefficient is necessary favorable for high $Z T .^{4}$ In view of the band structure of MnTe, the high Seebeck coefficient is intimately related to the fairly large band gap and the $p$ - $d$ overlap induced van Hove singularity in the electron density of states (DOS) near the valence band edge. ${ }^{22,23}$ Notably, this is in line with the "resonance level" scheme proposed by Heremans et al., which underlies an important approach to improve $Z T$ via enhancing the Seebeck coefficient. ${ }^{24}$

The main roadblock of MnTe for high TE performance is its high resistivity ( $\sim 1 \Omega \mathrm{cm}$ at room temperature) due to a low hole concentration $\left(\sim 10^{18} \mathrm{~cm}^{-3}\right.$ compared to the carrier concentration $10^{19}-10^{21} \mathrm{~cm}^{-3}$ in the state-of-the-art TE tellurides). ${ }^{17}$ To increase the carrier concentration, hole doping is apparently the option: not only it introduces additional hole charge carriers but also creates short-range defects that effectively scatter short wavelength heat-carry phonons at elevated temperatures. ${ }^{25}$ In this work, sulfur (S) was chosen as hole dopant for the following reasons: (1) Iso-electronic dopants with significantly different atomic mass $\left(\mathrm{M}_{\mathrm{Te}}=127.60 \mathrm{Da}\right.$ and $\left.\mathrm{M}_{\mathrm{S}}=32.066 \mathrm{Da}\right)$ will strengthen defect- and alloying-phonon scattering to suppress the lattice thermal conductivity. (2) According to Mahan and Sofo, ${ }^{26}$ the best TE performance is expected when the DOS is a $\delta$-function at $\sim 2.4 k_{\mathrm{B}} T$ above or below the Fermi level $\left(E_{\mathrm{F}}\right)$. In MnTe, the narrow Mn-3d orbital gives rise to a DOS singularity on top of a slow-varying Te-5p orbital-dominated background DOS. $^{22,23}$ The DOS spike is near the valence band edge. As such, iso-electronic doping is desired for a fine tune of the position of $E_{\mathrm{F}}$.

\section{EXPERIMENTS}

Manganese ingots (99.95\%, Alfa Aesar), tellurium lumps (99.999\%, Alfa Aesar), and sulfur powder $(99.5 \%$, Alfa Aesar) were weighted according to the nominal composition of $\mathrm{MnTe}_{1-\mathrm{x}} \mathrm{S}_{\mathrm{x}}(\mathrm{x}=0,0.05$, and 0.10$)$, placed into evacuated quartz tubes, heated up to $1173 \mathrm{~K}$ in $6 \mathrm{~h}$, and then homogenised for 1 week. Then, the samples were quenched in ice water. The obtained ingots were hand-pulverized followed by a spark plasma sintering (SPS) densification processing at $873 \mathrm{~K}$ for $8 \mathrm{~min}$ under a uniaxial pressure of $50 \mathrm{MPa}$.

Phase structure, chemical composition, and micromorphology of the $\mathrm{MnTe}_{1-\mathrm{x}} \mathrm{S}_{\mathrm{x}}$ samples were analyzed by means of X-ray diffraction (XRD) (PANalytical X'Pert Pro ${ }^{\circledR}$ $\mathrm{X}$-ray diffractometer), and field emission scanning electron microscopy (SEM) with energy-dispersive X-ray spectroscopy (EDS) option. The EDS results have been subjected to ZAF corrections (atomic number correction, absorption correction, and fluorescence correction). The Archimedes' method revealed that the packing densities $(d)$ of the SPSed pellets are $6.05 \mathrm{~g} / \mathrm{cm}^{3}, 5.98 \mathrm{~g} / \mathrm{cm}^{3}$, and $5.85 \mathrm{~g} / \mathrm{cm}^{3}$ for MnTe, $\mathrm{MnTe}_{0.95} \mathrm{~S}_{0.05}$, and $\mathrm{MnTe}_{0.9} \mathrm{~S}_{0.1}$, respectively. These densities are $>96 \%$ of the theoretical density. The thermal diffusivity was measured by the laser flash method on a Netzsch LFA $457^{\circledR}$ system from 300 to $773 \mathrm{~K}$. Specific heat was measured by differential scanning calorimetry on a Netzsch DSC- $404 C^{\circledR}$ calorimeter from 300 to $673 \mathrm{~K}$. The thermal conductivity, $\kappa$, was then calculated from the thermal

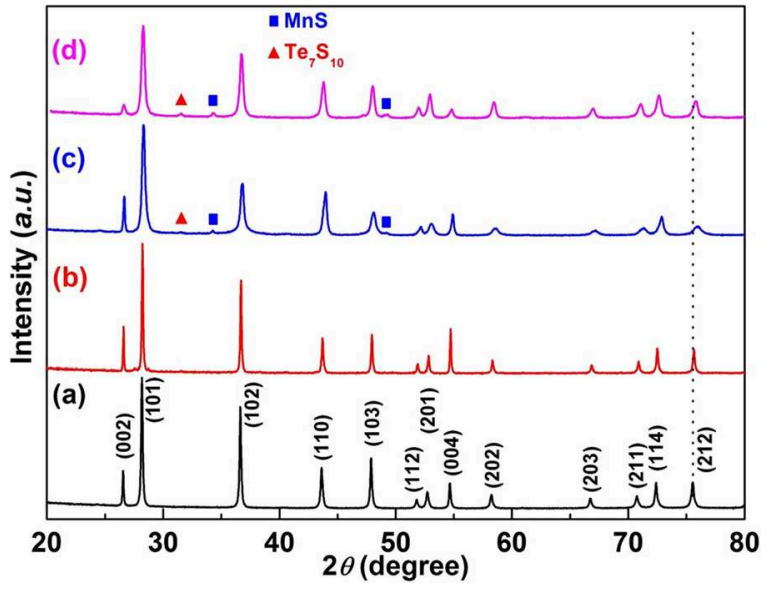

FIG. 1. XRD patterns of as-synthesized powder (a) MnTe, (b) $\mathrm{MnTe}_{0.95} \mathrm{~S}_{0.05}$, (c) $\mathrm{MnTe}_{0.9} \mathrm{~S}_{0.1}$, and (d) SPSed bulk sample of $\mathrm{MnTe}_{0.9} \mathrm{~S}_{0.1}$. The dashed line is to help visualize the shift of (212) peak.

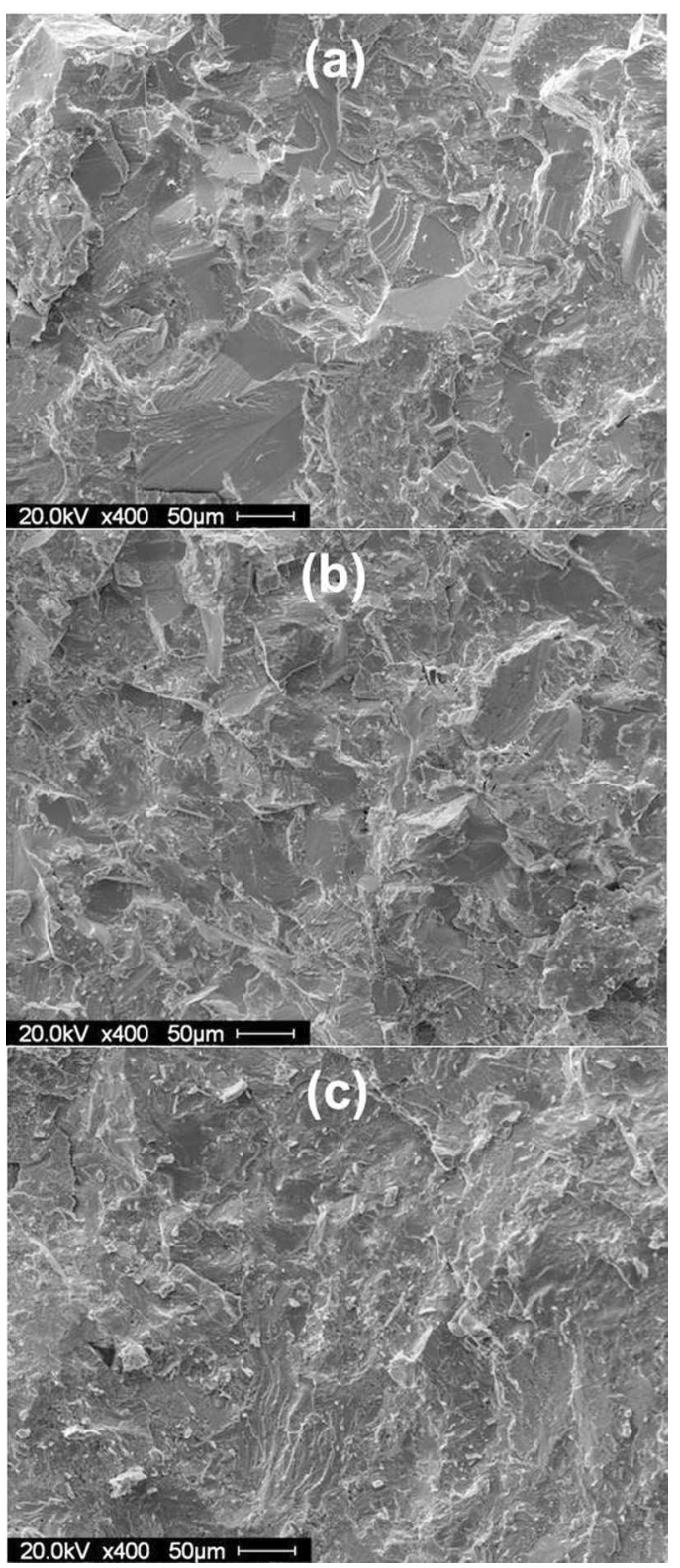

FIG. 2. SEM images of fracture surface on SPSed bulk samples: (a) MnTe, (b) $\mathrm{MnTe}_{0.95} \mathrm{~S}_{0.05}$, and (c) $\mathrm{MnTe}_{0.9} \mathrm{~S}_{0.1}$. 
diffusivity, $D$, specific heat per unit volume, $C_{\mathrm{p}}$, and density, $d$, via the relationship, $\kappa=D C_{\mathrm{p}} d$. After the thermal diffusivity measurement, the pellet samples were cut into $\sim 2.5 \times 2 \times 10 \mathrm{~mm}^{3}$ bars for electrical conductivity and Seebeck coefficient measurements on an Ozawa system under an Ar atmosphere from 300 to $773 \mathrm{~K}$. Hall coefficients, $R_{H}$, were measured on a Quantum Design Physical Properties Measurement System using a four-probe configuration by sweeping the magnetic field between $\pm 0.5 \mathrm{~T}$ from $5 \mathrm{~K}$ to $300 \mathrm{~K}$. The effective carrier concentration, $n$, and Hall mobility, $\mu$, were then calculated from the relations $n=1 / \mathrm{e} R_{H}$ and $\mu=\sigma / n e$, respectively, where $e$ is the elemental charge. The error bar for electrical conductivity, Seebeck coefficient, and thermal conductivity is $5 \%, 5 \%$, and $10 \%$, respectively.

\section{RESULTS AND DISCUSSION}

\section{A. Phase structure and microstructure}

XRD patterns of powder and pelletized $\mathrm{MnTe}_{1-\mathrm{x}} \mathrm{S}_{\mathrm{x}}(\mathrm{x}=0$, 0.05 , and 0.1 ) samples are displayed in Fig. 1. All diffraction peaks can be indexed to the NiAs structure with the peak position shifting systematically to higher angle with increasing $\mathrm{S}$ content. This is consistent with a decreasing lattice constant upon $\mathrm{S}$ doping on the Te site. Trace amounts of $\mathrm{MnS}$ and $\mathrm{Te}_{7} \mathrm{~S}_{10}$ are observed in the sample with high $\mathrm{S}$ content.

SEM images taken on the fracture surface of SPSed pellets are presented in Fig. 2. Despite an anisotropic crystal structure, the fracture surface does not show any sign of preferred orientation. In addition, the pellet is densely packed, consistent with the results of the Archimedes method. Elemental composition analysis is carried out using ZAFcorrected EDS. The results of point analysis and elemental mapping are shown in Figs. 3 and 4, respectively. The compositions of nominal MnTe, $\mathrm{MnTe}_{0.95} \mathrm{~S}_{0.05}$, and $\mathrm{MnTe}_{0.9} \mathrm{~S}_{0.1}$ are found to be $\mathrm{Mn}_{0.953} \mathrm{Te}, \mathrm{Mn}_{0.958} \mathrm{Te}_{0.981} \mathrm{~S}_{0.019}$, and $\mathrm{Mn}_{0.957} \mathrm{Te}_{0.978} \mathrm{~S}_{0.022}$, respectively. Despite a slight Mn deficiency, the Mn content remains the same for all three samples. It thus allows to correlate the S-doping effects on the thermoelectric properties via comparison. Notably, the measured $\mathrm{S}$ content is much lower than the nominal ratio, accompanied by the formation of $\mathrm{MnS}$ and $\mathrm{Te}_{10} \mathrm{~S}_{7}$ secondary phases (Fig. 4). The secondary phases are isolated in the matrix of the primary phase, and they presumably do not affect the transport properties given their small volume fractions. Importantly, the XRD patterns, SEM images, and EDS mapping results corroborate that $S$ uniformly substitutes Te on a length scale of $\mu \mathrm{m}$ and above.

\section{B. Electrical transport properties}

The temperature dependent electrical transport properties of all samples are shown in Fig. 5. As presented in Fig. 5(a), the electrical conductivity increases significantly with increase of $\mathrm{S}$ content. The electrical conductivity exhibits a plateau between room temperature and $\sim 500 \mathrm{~K}$, above which

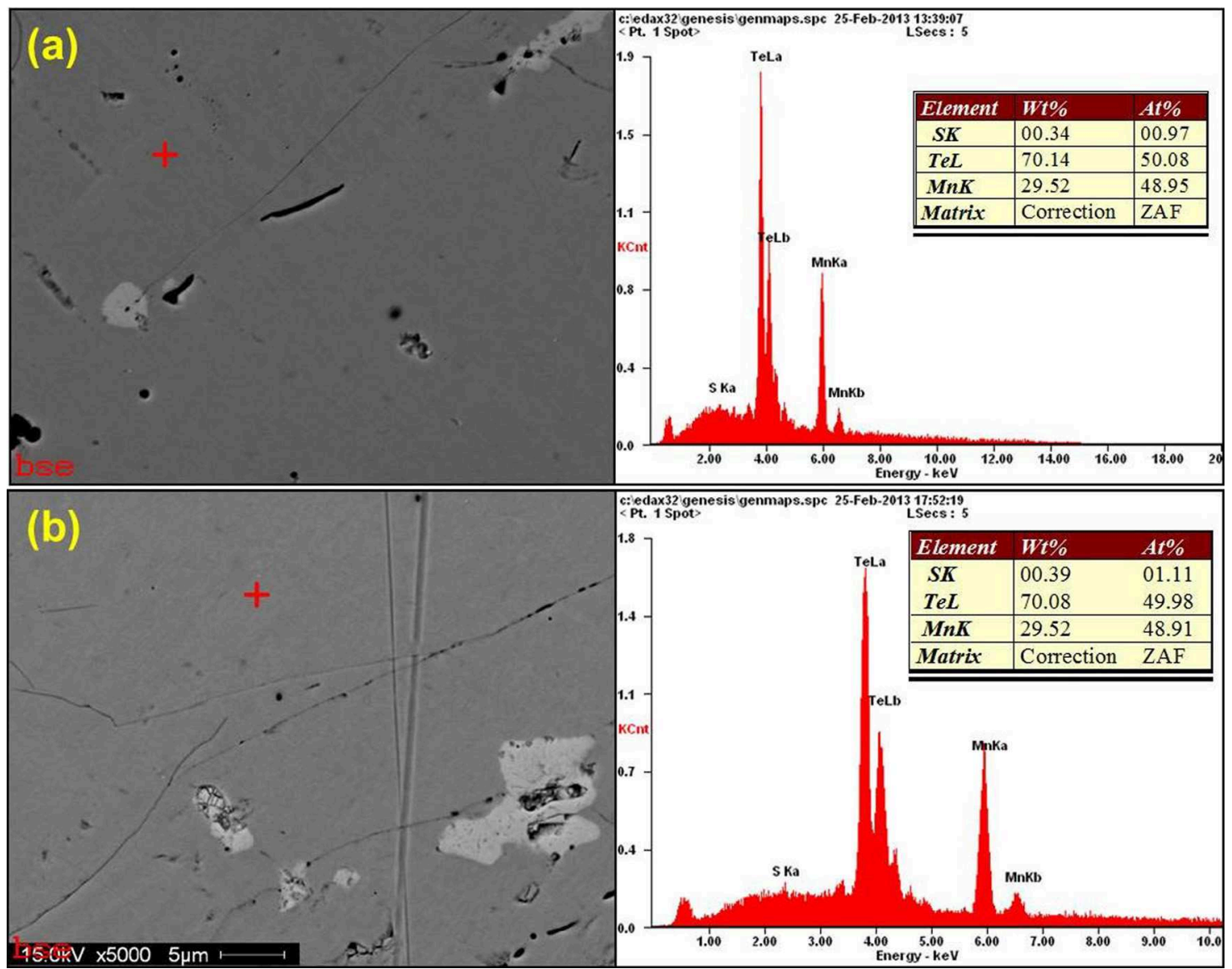

FIG. 3. Backscattered electron microscope images with the corresponding EDS analysis results of SPSed bulk materials: (a) $\mathrm{MnTe}_{0.95} \mathrm{~S}_{0.05}$ and (b) $\mathrm{MnTe}_{0.9} \mathrm{~S}_{0.1}$. 

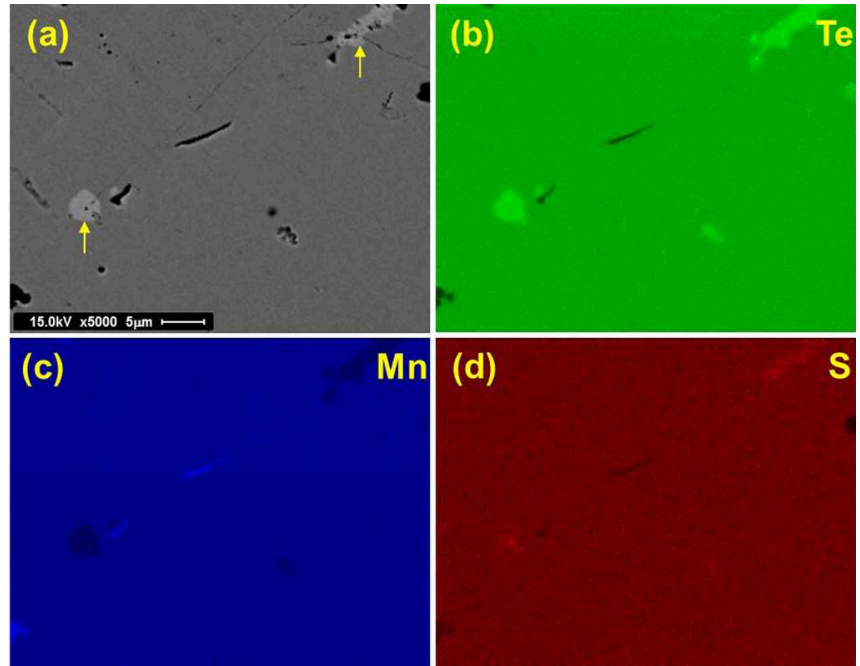

FIG. 4. EDS elemental mapping results of SPSed $\mathrm{MnTe}_{0.95} \mathrm{~S}_{0.05}$ bulk samples: (a) back scattering image, (b) Te, (c) Mn, and (d) S. The $\mathrm{Te}_{10} \mathrm{~S}_{7}$ secondary phase in (a) is indicated by arrows.
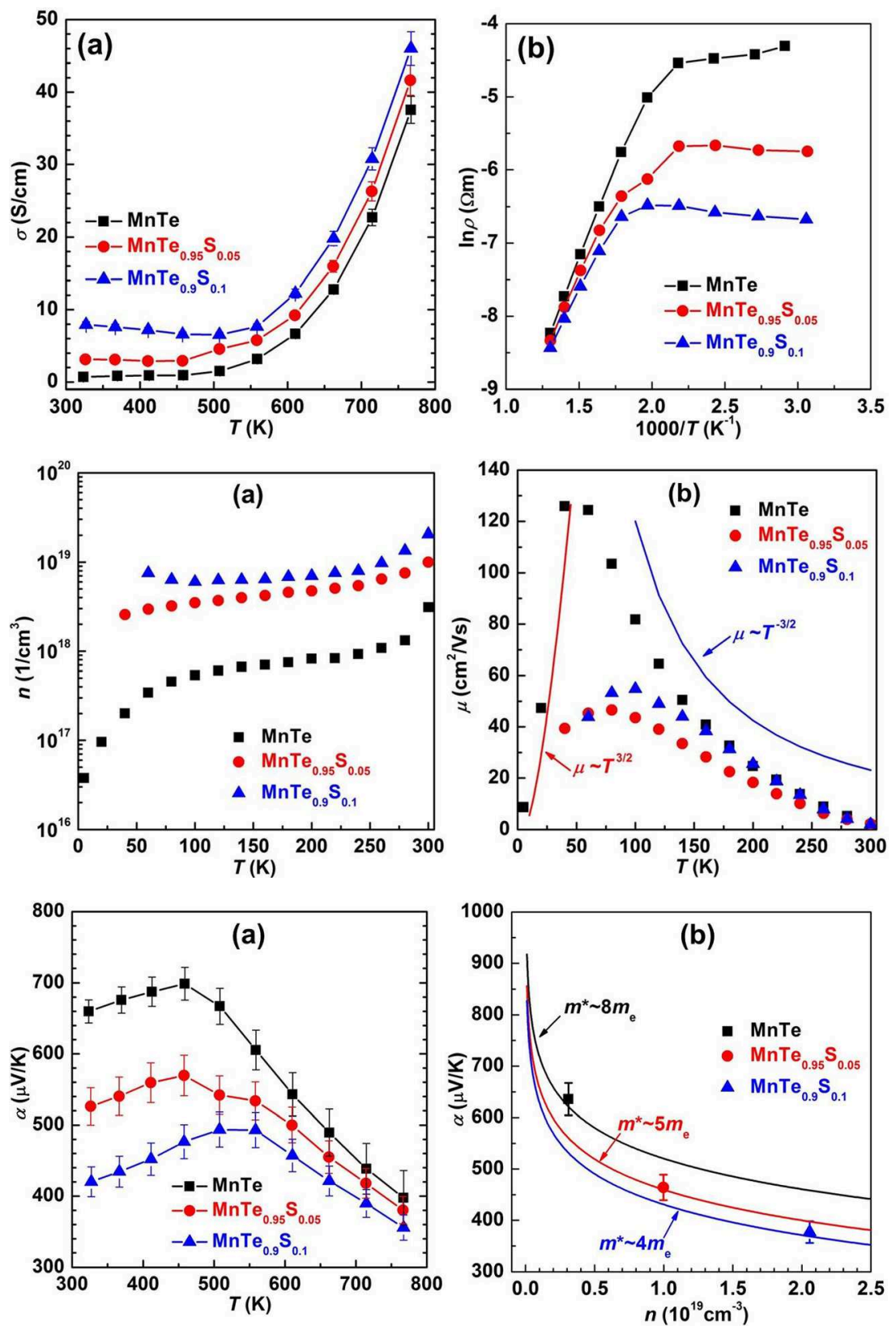

it rapidly increases with increasing temperature. The plateau can be attributed to the exhaustion range and the fast increasing regime to the intrinsic excitation regime. For a quantitative analysis, the band gap $\left(E_{\mathrm{g}}\right)$ can be extracted from the slope of $\ln \rho$ vs. 1000/T curve (Fig. 5(b)) using Arrhenius formula

$$
\rho=\rho_{0} \exp \left(E_{g} / 2 k_{B} T\right)
$$

where $\rho$ is the electrical resistivity $(\rho=1 / \sigma)$, and $\rho_{0}$ a constant. The calculated intrinsic band gap values are $\sim 0.89 \mathrm{eV}$, $0.77 \mathrm{eV}$, and $0.68 \mathrm{eV}$ for $\mathrm{MnTe}, \mathrm{MnTe}_{0.95} \mathrm{~S}_{0.05}$, and $\mathrm{MnTe}_{0.9} \mathrm{~S}_{0.1}$, respectively. The obtained band gap value of pristine MnTe is very close to the theoretical result $(\sim 0.86 \mathrm{eV}){ }^{9}$

To further understand the mechanism(s) underlying the electrical conductivity enhancement, Hall coefficient measurements was measured and the results are presented in Fig. 6. As clearly shown in Fig. 6(a), the carrier (hole)
FIG. 5. (a) Electrical conductivity of SPSed $\mathrm{MnTe}_{1-\mathrm{x}} \mathrm{S}_{\mathrm{x}}$ bulk samples and (b) $\ln \rho$ vs $1000 / T$ plot.
FIG. 6. Temperature dependence of (a) carrier concentration and (b) carrier mobility of SPSed $\mathrm{MnTe}_{1-\mathrm{x}} \mathrm{S}_{\mathrm{x}}$ bulk samples.
FIG. 7. (a) Temperature dependence of the Seebeck coefficient and (b) $\alpha-n$ plot for $\mathrm{MnTe}_{1-\mathrm{x}} \mathrm{S}_{\mathrm{x}}$ compounds; solid line in (b) represents the Pisarenko relation with different $m^{*}$. 
concentration significantly increases with an increase of the $\mathrm{S}$ content. At room temperature, the hole concentration for MnTe, $\mathrm{MnTe}_{0.95} \mathrm{~S}_{0.05}$, and $\mathrm{MnTe}_{0.9} \mathrm{~S}_{0.1}$ is $3.0 \times 10^{18} \mathrm{~cm}^{-3}$, $1.0 \times 10^{19} \mathrm{~cm}^{-3}$, and $2.1 \times 10^{19} \mathrm{~cm}^{-3}$, respectively. Apparently, $\mathrm{S}$ is an effective hole dopant since it generates acceptor levels near the top of valence band. Furthermore, the temperature variation of the carrier concentration confirms our attribution of the characteristic regimes in the electrical conductivity to the exhaustion regime and the intrinsic excitation regime. The calculated temperature dependence of the Hall mobility is shown in Fig. 6(b). As compared to the pristine sample, the S-doping induced short-range defects strongly scatter the charge carriers at temperatures below $100 \mathrm{~K}$. Hall mobility follows a $T^{3 / 2}$ dependence as expected from ionized impurity scattering. At elevated temperatures, the conventional electron-acoustic phonon coupling gradually takes over: the temperature dependence of the Hall mobility crosses over to a $T^{-3 / 2}$ behavior. Note that the Hall mobilities of all three samples are nearly the same in the vicinity of room temperature.

The temperature dependent Seebeck coefficients are displayed in Fig. 7(a). The signs of the Seebeck coefficient and of the Hall coefficient are positive in the temperature range studied, confirming a $p$-type conduction. In addition, the magnitude of the Seebeck coefficient decreases systematically with an increase of the $\mathrm{S}$ content. The Seebeck coefficient first increases with temperature, peaks at a certain temperature, and then decreases at elevated temperatures. The peak position coincides with the onset of the intrinsic excitation regime (Fig. 5(a)). In the intrinsic excitation regime, the electron-hole pairs are thermally excited, electrons (minority carriers) and holes (majority carriers) cancel out in the Seebeck coefficient, leading a maximum. Therefore, the Seebeck peak signatures the onset of the bipolar effect.

A single parabolic band (SPB) model analysis is conducted. Such a SPB model appears oversimplified considering the actual electronic band structure of MnTe, nonetheless, such an analysis helps to gain more insight into the sulfur-doping effects on the microscopic transport parameters, and it provides semi-quantitative guidance for further doping study. In the limit of a non-degenerate electron gas, the Seebeck coefficient in a SPB model with single relaxation time approximation is related to the carrier density $n$ and scattering parameter $\lambda$ via

$$
\begin{aligned}
\alpha & =\frac{k_{\mathrm{B}}}{e}(2+\lambda-\eta) \\
& =\frac{k_{\mathrm{B}}}{e}\left\{2+\lambda+\ln \left[\frac{2\left(2 \pi m^{*} k_{\mathrm{B}} T / h^{2}\right)^{3 / 2}}{n}\right]\right\},
\end{aligned}
$$

where $\eta$ is the reduced Fermi energy, $\lambda$ the energy-dependent scattering parameter, and $\lambda=0,2$ for acoustic phonon scattering and ionized impurity scattering, respectively, $m^{*}$ the carrier effective mass, and $h$ the Planck constant. ${ }^{25,27}$ Since the temperature dependence of mobility between $100 \mathrm{~K}$ and $300 \mathrm{~K}$ follows a $T^{-3 / 2}$ behavior (Fig. 6(b)), it is plausible to assume that the acoustic phonon scattering is the dominant scattering mechanism above room temperature. Therefore, $\lambda=0$ was chosen to calculate $m^{*}$ via Eq. (2). By using the carrier concentration and Seebeck coefficient of $\mathrm{MnTe}_{1-\mathrm{x}} \mathrm{S}_{\mathrm{x}}$ at room temperature, the $m^{*}$ of $\sim 8 m_{\mathrm{e}}, \sim 5 m_{\mathrm{e}}$, and $\sim 4 m_{\mathrm{e}}$ are obtained for MnTe, $\mathrm{MnTe}_{0.95} \mathrm{~S}_{0.05}$, and $\mathrm{MnTe}_{0.9} \mathrm{~S}_{0.1}$, respectively. The Pisarenko plot (Fig. 7(b)) suggests that $\mathrm{S}$ doping not only increases the carrier concentration but also lowers the effective mass. The Seebeck coefficient decreasing with increase of the sulfur content is attributed to the hole concentration increase and the effective mass decrease. This
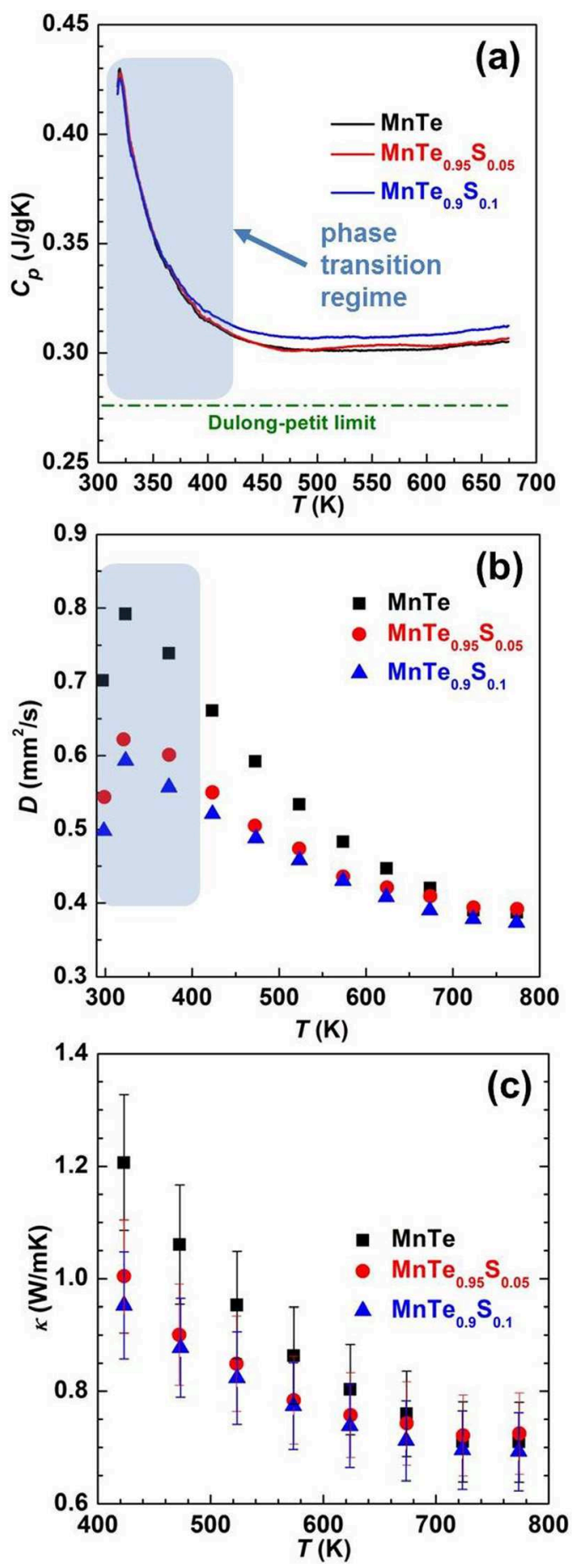

FIG. 8. Temperature dependence of thermal transport properties for SPSed $\mathrm{MnTe}_{1-\mathrm{x}} \mathrm{S}_{\mathrm{x}}$ bulk samples: (a) specific heat; (b) thermal diffusivity; and (c) thermal conductivity. The mark area in (a) and (b) indicates the phase transition regime, and the calculated thermal conductivity is only presented above the phase transition regime in (c). 

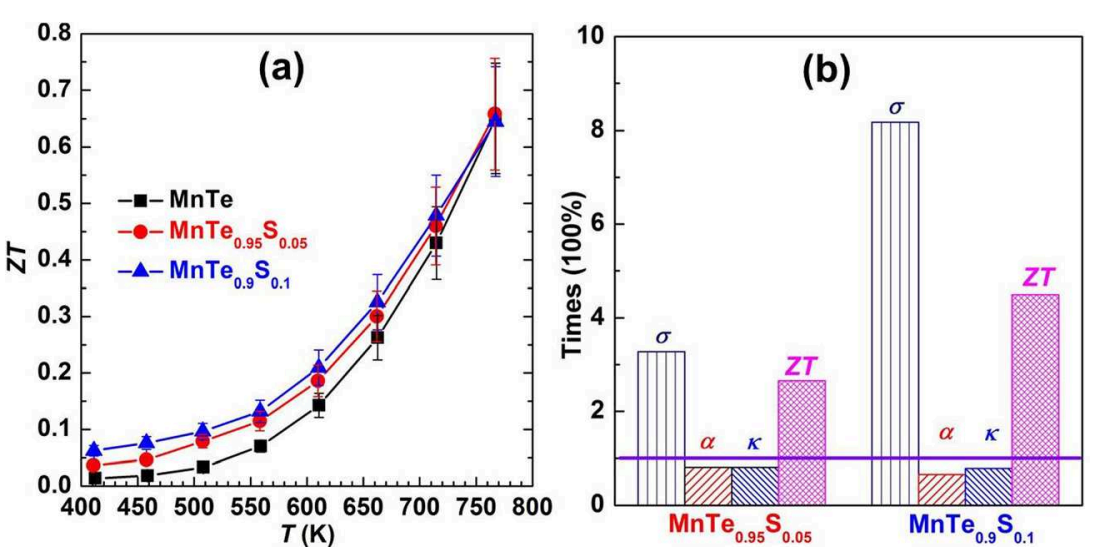

FIG. 9. (a) The figure of merit, $Z T$, of SPSed $\mathrm{MnTe}_{1-\mathrm{x}} \mathrm{S}_{\mathrm{x}}$ bulk samples. (b) Resulting enhancement of $Z T$ for $\mathrm{MnTe}_{0.95} \mathrm{~S}_{0.05}$ and $\mathrm{MnTe}_{0.9} \mathrm{~S}_{0.1}$ at $410 \mathrm{~K}$ compared with the reference (pristine MnTe). The $\sigma, \alpha, \kappa$, and $Z T$ of $\mathrm{MnTe}_{0.95} \mathrm{~S}_{0.05}$ and $\mathrm{MnTe}_{0.9} \mathrm{~S}_{0.1}$ are normalized by the corresponding values of the pristine MnTe (the solid tie line denotes the reference). The estimated error for $Z T$ is $\pm 15 \%$ according to the uncertainties of electrical conductivity, the Seebeck coefficient, and thermal conductivity. effective mass decrease is thermoelectrically detrimental, and how to mitigate this effect will be the main subject of future studies.

\section{Thermal transport properties}

The temperature dependent specific heat $\left(C_{\mathrm{p}}\right)$, thermal diffusivity $(D)$, and thermal conductivity $(\kappa)$ of $\mathrm{MnTe}_{1-\mathrm{x}} \mathrm{S}_{\mathrm{x}}$ samples are presented in Fig. 8. $C_{\mathrm{p}}$ exhibits a peak around $320 \mathrm{~K}$ (Fig. 8(a)), $D$ has a feature near the same temperature (Fig. 8(b)), both features are attributed to a known antiferromagnetic-paramagnetic transition. ${ }^{28,29}$ It is particularly interesting that $C_{\mathrm{p}}$ at high temperatures is substantially higher than the Dulong-Petit limit [dashed line in Fig. 8(a)], which can be attributed to anharmonicity and/or magnons at elevated temperatures. The thermal conductivity is calculated by the relationship $\kappa=D C_{\mathrm{p}} d,^{30}$ and the result is displayed in Fig. 8(c). Due to low electrical conductivity, the total thermal conductivity is dominated by the lattice contribution. Owing to the alloy scattering and mass fluctuation scattering, the thermal conductivity of $\mathrm{MnTe}_{1-\mathrm{x}} \mathrm{S}_{\mathrm{x}}$ samples decreases substantially with the increase of $S$ below $600 \mathrm{~K}$. For example, the thermal conductivity of $\mathrm{MnTe}_{0.9} \mathrm{~S}_{0.1}$ sample is decreased by $20 \%$ at $410 \mathrm{~K}$ compared with that of the pristine MnTe sample. At higher temperatures, the bipolar effect becomes prominent, and the S-doping does not make much difference in the thermal conductivity.

\section{The dimensionless figure of merit ZT}

The dimensionless figures of merit ZT of the $\mathrm{MnTe}_{1-\mathrm{x}} \mathrm{S}_{\mathrm{x}}$ samples are shown in Fig. 9(a) as a function of temperature. Due to the significantly improved electrical conductivity and suppressed thermal conductivity, the $Z T$ of the $\mathrm{MnTe}_{0.9} \mathrm{~S}_{0.1}$ samples is 7 times higher than that of MnTe sample at $410 \mathrm{~K}$. Fig. 9(b) shows the various properties of the $\mathrm{MnTe}_{0.95} \mathrm{~S}_{0.05}$ and $\mathrm{MnTe}_{0.9} \mathrm{~S}_{0.1}$ samples normalized by their counterparts. However, the improvement of $Z T$ gets marginal at elevated temperatures: the $Z T$ values of all three $\mathrm{MnTe}_{1-\mathrm{x}} \mathrm{S}_{\mathrm{x}}$ samples are $\sim 0.65$ at $773 \mathrm{~K}$, the maximum temperature attained in this work.

\section{CONCLUSIONS}

In this work, $\mathrm{MnTe}_{1-\mathrm{x}} \mathrm{S}_{\mathrm{x}}$ compounds were prepared by melt-quenching method followed by pulverization and spark plasma sintering processing. The effects of iso-electronic $\mathrm{S}$ doping on the electrical and thermal transport properties of $\mathrm{MnTe}_{1-\mathrm{x}} \mathrm{S}_{\mathrm{x}}$ were systematically investigated. In general, $\mathrm{S}$ doping led to two thermoelectrically favorable changes in the carrier concentration and in the thermal conductivity but also one thermoelectrically unfavorable change in the effective mass. As a result, the $Z T$ of $\mathrm{MnTe}_{0.9} \mathrm{~S}_{0.1}$ samples was 7 times higher than that of MnTe sample at $410 \mathrm{~K}$, mainly due to a significantly improved electrical conductivity. The improvement of $Z T$ got marginal at elevated temperatures: all three samples attained a $Z T$ value $\sim 0.65$ at $773 \mathrm{~K}$. Future work will focus on tuning the Fermi level by finer doping while minimizing the Seebeck coefficient degradation.

\section{ACKNOWLEDGMENTS}

W.J.X. would like to thank the Marie Curie COFUND fellowship supported by EU FP7 and Empa. The work at Empa was partially supported by DFG SPP1386 and Swisselectric. Y.F.L. and J.H. would like to thank the support of NSF DMR-1307740.

${ }^{1}$ T. M. Tritt and M. A. Subramanian, MRS Bull. 31, 188 (2006).

${ }^{2}$ M. S. Dresselhaus, G. Chen, M. Y. Tang, R. G. Yang, H. Lee, D. Z. Wang, Z. F. Ren, J. P. Fleurial, and P. Gogna, Adv. Mater. 19, 1043 (2007).

${ }^{3}$ J. R. Sootsman, D. Y. Chung, and M. G. Kanatzidis, Angew. Chem., Int. Ed. 48, 8616 (2009).

${ }^{4}$ T. M. Tritt, Annu. Rev. Mater. Res. 41, 433 (2011).

${ }^{5}$ G. J. Snyder and E. S. Toberer, Nature Mater. 7, 105 (2008).

${ }^{6}$ G. Zanmarchi and C. Haas, Philips Res. Rep. 23, 389 (1968).

${ }^{7}$ J. D. Wasscher, Philips Res. Rep., Suppl. 1 (1969).

${ }^{8}$ W. Szuszkiewicz, E. Dynowska, B. Witkowska, and B. Hennion, Phys. Rev. B 73, 104403 (2006).

${ }^{9}$ M. Sandratskrii, R. F. Egorovan, and A. A. Berdyshev, Phys. Status Solidi B 104, 103 (1981).

${ }^{10}$ J. O. Sofo and G. D. Mahan, Phys. Rev. B 49, 4565 (1994).

${ }^{11}$ J. He, Y. Liu, and R. Funahashi, J. Mater. Res. 26, 1762 (2011).

${ }^{12}$ B. Hennion, W. W. Szuszkiewicz, E. Dynowska, E. Janik, and T. Wojtowicz, Phys. Rev. B 66, 224426 (2002).

${ }^{13}$ Y. B. Li, Y. Q. Zhang, N. K. Sun, Q. Zhang, D. Li, J. Li, and Z. D. Zhang, Phys. Rev. B 72, 193308 (2005).

${ }^{14}$ J. B. C. Efrem D'Sa, P. A. Bhobe, K. R. Priolkar, A. Das, S. K. Paranjpe, R. B. Prabhu, and P. R. Sarode, J. Magn. Magn. Mater. 285, 267 (2005).

${ }^{15}$ S. S. Aplesnin, L. I. Ryabinkina, O. B. Romanova, D. A. Balaev, O. F. Demidenko, K. I. Yanushkevich, and N. S. Miroshnichenko, Phys. Solid State 49, 2080 (2007).

${ }^{16}$ X. He, Y. Q. Zhang, and Z. D. Zhang, J. Mater. Sci. Technol. 27, 64 (2011).

${ }^{17}$ Y. Magnin and H. T. Diep, Phys. Rev. B 85, 184413 (2012). 
${ }^{18}$ Ch. Ferrer-Roca, A. Segura, C. Reig, and V. Munoz, Phys. Rev. B 61, 13679 (2000).

${ }^{19}$ B. Kim, I. Kim, B. Min, M. Oh, S. Park, and H. Lee, Electron. Mater. Lett. 9, 477 (2013).

${ }^{20}$ W. J. Xie, X. F. Tang, Y. G. Yan, Q. J. Zhang, and M. T. Tritt, Appl. Phys. Lett. 94, 102111 (2009).

${ }^{21}$ W. J. Xie, J. He, H. J. Kang, X. F. Tang, S. Zhu, M. Laver, S. Y. Wang, J. Copley, C. Brown, Q. J. Zhang, and T. M. Tritt, Nano Lett. 10, 3283 (2010).

${ }^{22}$ J. W. Allen, G. Lucovsky, and J. C. Mikkelsen, Jr., Solid State Commun. 24, 367 (1977).

${ }^{23}$ S. J. Youn, B. I. Min, and A. J. Freeman, Phys. Status Solidi B 241, 1411 (2004).

${ }^{24}$ J. P. Heremans, B. Wiendlocha, and A. M. Chamoire, Energy Environ. Sci. 5, 5510 (2012).

${ }^{25}$ A. F. Ioffe, Semiconductor Thermoelements, and Thermoelectric Cooling (Infosearch, London, 1957).
${ }^{26}$ G. D. Mahan and J. O. Sofo, Proc. Natl. Acad. Sci. U.S.A. 93, 7436 (1996).

${ }^{27}$ A. F. May and G. J. Snyder, "Introduction to modeling thermoelectric transport at high temperature" in Materials, Preparation, and Characterization in Thermoelectrics (CRC Press, Boca Raton, FL, 2012).

${ }^{28}$ J. J. Banewicz, R. F. Heidelberg, and A. H. Luxem, J. Phys. Chem. 65, 615 (1961).

${ }^{29}$ Z. H. Wang, D. Y. Geng, W. J. Gong, J. Li, Y. B. Li, and Z. D. Zhang, Thin Solid Films 522, 175 (2012).

${ }^{30} \mathrm{We}$ cut off the $C_{\mathrm{p}}$ data in the temperature range of $300-400 \mathrm{~K}$, i.e., the phase transition regime, as the DSC does not give the correct reading of $C_{\mathrm{p}}$ through a phase transition. A guarded hot plate method is more appropriate. Nonetheless, this would not alter the following discussion as our main focus is the $Z T$ value at a temperature much high than the Néel temperature. Besides, the $C_{\mathrm{p}}$ data above $673 \mathrm{~K}$ are obtained from linear extrapolation of the measurement data (300-673 K). 